\title{
Der Einfluß von Vitamin $K$ auf Gerinnungsfaktoren und Enzyme mit kurzer Halbwertszeit in der Rattenleber
}

\author{
Von E. F. Vogeler ${ }^{1}$ ), H. Reinauer und S. Hollmand \\ Institut für physiologische Chemie I der Universität Diisseldorf
}

(Eingegangen am 29. November 1971)

Ratten wurden durch tägliche Injektionen von Marcumar in einen Vitamin K-Mangelzustand gebracht. In Normal- und Mangeltieren wurde die Aktivität der Gerinnungsfaktoren II und V sowie die Aktivität der Enzyme Tryptophan-Pyrrolase (EC 1.13.1.12), Serin-Dehydratase (EC 4.2.1.13) und Aldolase (EC 4.1.2.13) gemessen. Im Vitamin K-Mangel zeigten die Gerinnungsfaktoren und die Enzyme bis auf die Aldolase einen signifikanten Abfall gegenüber den Kontrolltieren. Injektionen von Menadion an Vitamin K-Mangeltiere ließ die Aktivität der Tryptophan-Pyrrolase und des Prothrombins über den Kontrollwert hinaus ansteigen. Dicser Effekt war sowohl durch Actinomycin $\mathrm{D}$ als auch durch Cycloheximid hemmbar. Aufgrund dieser Ergebnisse wird einc unspezifische Wirkung von Vitamin $\mathrm{K}$ auf die Synthese von Enzymen mit kurzer Halbwertszeit abgeleitct. Als Wirkort wird neben der Energiebereitstellung eine unspezifische Beeinflussung der ribosomalen Proteinsynthese diskutiert. Auf die klinische Bedeutung der erhobenen Befunde wird hingewiesen.

\section{The influence of vitamin $K$ on clotting factors and enzymes with short balf lives in rat liver}

Rats were made vitamin $\mathrm{K}$ deficient by daily injections of marcumar. The activities of clotting factors II and V and the activities of the enzymes tryptophan pyrrolase, serine dehydratase and aldolase were measured in normal and deficient animals. In K-deficient animals, the clotting factors and the enzymes, except aldolase, showed a significant decrease comparcd to control animals. After the injection of menadione into the $\mathrm{K}$-deficient animals, the activities of tryptophan pyrrolase and prothrombin rose above those of the controls. This increase was inhibited by actinomycin $\mathrm{D}$ or cycloheximide. These results indicate that vitamin $\mathrm{K}$ has a non-specific action on the synthesis of enzymes with short half lives. In addition to its role in energy metabolism, vitamin $\mathrm{K}$ appears to influence non-specifically the ribosomal protein synthesis. The clinical importance of these findings is discussed.

In zahlreichen Experimenten wurde festgestellt, daß Gaben von Vitamin $K$ die Aktivität der Gerinnungsfaktoren (II, VII, IX, X) im Plasma und in der Leber von Vögeln und Säugetieren erhöhen, bei denen ein Vitamin K-Mangel entweder durch Mangeldiät oder durch Warfarin- oder Dicumarol-Gabe hervorgerufen wurde. Diese Befunde wurden an der isolierten perfundierten Rattenleber bestätigt $(1-6)$. Der aktivierende Einfluß von Vitamin $K$ auf die Prothrombinsynthese im Vitamin K-Mangel konnte mit Hemmstoffen der Proteinsynthese (Äthionin, Actinomycin D, Puromycin, Cycloheximid) unterbunden werden. Aufgrund von früheren Beobachtungen an Vitamin $\mathrm{K}$ Mangel-Hühnern vermutete man den Wirkungsort des Vitamin $\mathrm{K}$ bei der Transscription $(7,8)$. Spätere Untersuchungen schrieben dem Vitamin $\mathrm{K}$ eine Kontrollfunktion im Bereich der ribosomalen Proteinsynthese zu $(5,9,10)$. Andere Untersucher suchten die Wirkung bei der Ablösung des fertigen Prothrombinmoleküls von der messenger-RNA $(4,11)$.

Entsprechend den vorliegenden Befunden käme dem Vitamin $\mathrm{K}$ eine spezifische Bedeutung für die Prothrombinsynthese zu. Berücksichtigt man die biologische Halbwertszeit der von Vitamin $\mathrm{K}$ gesteuerten Gerinnungsfaktoren, so fällt auf, daß die Syntheseund die Abbauraten kurz sind (12-14).

1) Die vorliegende Atbeit enthält wesentliche Teile der Dissertation von E. F. Vogeler.
In der vorliegenden Arbeit soll im Tierversuch geklärt werden, ob im Vitamin K-Mangel neben den Gerinnungsfaktoren auch die Aktivitäten von Enzymen mit kurzer Halbwertszeit verändert sind, um auf diese Weise das Wirkungsspektrum und die Wirkungsspezifität des Vitamins zu erfassen.

\section{Material und Methodik}

\section{Reagenzien}

Marcumar [3-(1-Phenylpropyl)-4-hydroxycumarin, Hoffmann-La Roche AG, Grenzach; Faktor II bzw. Faktor V Testbesteck, Behringwerke AG, Marburg; für die Eichkurve der BiuretMethode wurde Serum-Albumin vom Rind (Behringwerke, Charge A 651) benutzt; Glycerin-3-phosphat-Dehydrogenase/ Triosephosphat-Isomerase aus Kaninchenmuskel (EC 1.1.1.8/ EC 5.3.1.1) und Lactatdehydrogenase aus Schweineherz (EC 1.1.1.27), Boehringer, Mannheim; Pyridoxal-5-phosphat, Merck AG, Darmstadt; Actinomycin D und Cycloheximid, Serva Entwicklungslabor, Heidelberg; Menadion [2-Methyl-naphthochinon(1,4)], Merck AG, Darmstadt.

\section{Versucbstiere}

Männliche Albino-Ratten vom Stamm Wistar II (Tierzüchterei Brünger, Bokel), Gewicht 250-270 g, bekamen vor der Behandlung Standardfutter (Firma Höveler, Immigrath) und Wasser ad libitum. Für die Dauer der Behandlung wurde jegliches Futter entzogen.

Im Vorversuch wurcle die geeignete Dosis von Marcumar ermittelt.

Bei i. p.-Gabe von $500 \mu \mathrm{g}$ Marcumar/kg Körpergewicht traten bei den Ratten erst nach der vierten Injektion Blutungen der Schleimhäute auf, obwohl dcr Quickwert schon am zwvciten Tag kleiner 
als $10 \%$ war. Dic Überlebenszeit betrug nie länger als sechs Tage.

\section{Durclffillsrung der Versuche}

Im Hauptversuch wurde ein Teil der Tiere drei-Tage lang mit Marcumar (Dosierung: je $500 \mu \mathrm{g} / \mathrm{kg}$ Körpergewicht und Tag) behandelt und am vierten Tage getötet.

Die Kontrolltiere erhielten gleichzeitig mit den Mangeltieren physiol. $\mathrm{NaCl}-\mathrm{Lösung}$ intraperitoneal injiziert.

Am vierten Tage wurde sowobl den gespritzten als auch den scheingespritzten Ratten in einer Äthernarkose durch Aortenpunktion Blut entnommen und gleichzeitig die Leber entfernt. Die Leberproben wurden kurz in physiol. $\mathrm{NaCl}$-Lösung gewaschen und in flüssigem Stickstoff eingefroren. Die Aktivitätsmessung erfolgte entweder sofort oder am folgenden Tag. Unterschiede gegenüber sofortigen Bestimmungen konnten nicht festgestellt werden (27). Abweichungen traten erst nach mehrmaligem Auftauen und Einfrieren der Proben auf. Zur Aufarbeitung wurden die Leberproben gewogen, 1:5 (für Aldolase-, SerinDehydratase-, Eiweiß-Bestimmung) bzw. 1:7 (für TryptophanPyrrolase-Bestimmung) mit eiskaltem KCl-EDTA-Tris-Puffer $(0,15 \mathrm{M}-1 \mathrm{~mm}-0,1 \mathrm{~m} ; \mathrm{pH}=7,6)$ verdünnt, und zwei Min. lang mit dem Potrer-ElveHJEM-Homogenisator aufgeschlossen. Die 1:5 Verdünnung wurde in einer präparativen Ultrazentrifuge bei $100000 \mathrm{~g} 60 \mathrm{Min}$. lang zentrifugiert. Im Überstand wurde das Gesamteiweiß, die Aktivität der Aldolase und der Serin-Dehydratase gemessen. Die Gerinnungsfaktoren II und V wurden nach dem Einphasenprinzip nach Quick bestimmt (2). Bei dieser Methode wird dem Citratplasma gleichzeitig mit der Recalcifizierung eine optimale Menge Gewebsthrombokinase zugegeben. Die resultiexende Gerinnungszeit ist dann in weitem Konzentrationsbereich nur von den im zugefügten Plasma enthaltenen Faktoren abhängig, da alle übrigen Faktoren in optimaler Konzentration im Substrat enthalten sind (22).

Das Gesamteiweiß des Leberhomogenats wurde nach der BiuretMethode bestimmt (23), und die Aldolase nach Bruns (24). Die Aktivitätsbestimmung der Serin-Dehydratase erfolgte mit uniresentlicher Modifikation nach Prror und PrIEs $(25,26)$, und die der Tryptophan-Pyrrolase nach KNox und Auerbacr (17).

\section{Ergebnisse}

\section{Wirkung von Dicumarol auf Normaltiere}

In Tabelle 1 sind die Ergebnisse von 46 behandelten Ratten zusammengefaßt. Davon erhielten. 27 Tiere über drei Tage je $500 \mu \mathrm{g}$ Marcumar/kg Körpergewicht intraperitoneal injiziett und wurden am vierten Tag getötet. Die restlichen 19 Tiere dienten als Kontrolle und wurde mit physiol. NaCl-Lösung behandelt. Es zeigte sich, daß die Gerinnungsfaktoren II und V innerhalb von vier Tagen stark absanken. So lag bei

Tab. 1

Aktivität der Gerinnungsfaktoren im Blut und der Enzyme in der Leber von Normaltieren und Vitamin K-Mangeltieren (Werte: $\bar{x} \pm s_{x} ; P$ )

\begin{tabular}{|c|c|c|c|}
\hline Enzym & $\begin{array}{c}\text { Normal } \\
(\mathrm{n}=19)\end{array}$ & $\begin{array}{l}\text { Vitamin K- } \\
\text { Mangel } \\
(n=27)\end{array}$ & $\mathbf{P}$ \\
\hline $\begin{array}{l}\text { Faktor } 11 \\
\text { (Prothrombin) (\%) } \\
\text { Faktor V }\end{array}$ & $99,37 \pm 7,58$ & $3,19 \pm 0,82$ & $<0,001$ \\
\hline $\begin{array}{l}\text { Faktor } \mathrm{V} \text {. } \\
\text { (Accelerin) (\%) }\end{array}$ & $99,52 \pm 6,85$ & $62,11 \pm 6,89$ & $<0,001$ \\
\hline $\begin{array}{l}\text { Tryptophan-Pyrrolase } \\
\text { ( } \mu \text { Mol Kynurenin/g } \\
\text { Protein-Std.) }\end{array}$ & $15,12 \pm 1,41$ & $12,63 \pm 1,24$ & $<0,001$ \\
\hline $\begin{array}{l}\text { Serin-Dehydratase } \\
\text { ( } \mu \text { Mol/g Protein - Std.) } \\
\text { Aldolase }\end{array}$ & $7,11 \pm 1,01$ & $6,28 \pm 0,82$ & $<0,001$ \\
\hline$(\mu$ Mol $/ g$ Protein $\cdot$ Std. $)$ & $91,65 \pm 3,54$ & $89,83 \pm 3,35$ & $>0,5$ \\
\hline
\end{tabular}

Marcumar-Tieren nach drei Tagen das Prothrombin bei $3 \%$ und Faktor $\mathrm{V}$ bei $62 \%$ der Kontrollen. Auch die Aktivitäten der Tryptophan-Pyrrolase und SerinDehydratase zeigten zwar einen geringen aber trotzdem signifikanten Abfall $(P<0,001)$. Dagegen war die Differenz der Aldolase-Aktivität nicht signifikant $(P<0,5)$.

\section{Wirkung von Menadion auf Vitamin K-Mangel-Ratten}

24 Marcumar-Vitamin K-Mangelratten und Kontrolltiere erhielten Menadion (100 $\mathrm{mg} / \mathrm{kg}$ Körpergewicht) intraperitoneal injiziert. Nach jeweils 2, 4, 6, 8 und 10 Stdn. wurden die Tiere getötet, im Blut die Prothrombinzeit und im Leberhomogenat das Gesamteiweiß und die Aktivität der Tryptophan-Pyrrolase bestimmt.

In Tabelle 2 ist die Aktivität der Tryptophan-Pyrrolase. und des Prothrombins in Abhängigkeit von der Zeit dargestellt. Vergleicht man die Ergebnisse mit den Werten bei Vitamin K-Mangel, so zeigt sich bei der

Tab. 2

Aktivität der Tryptophan-Pyrrolase in der Leber und ProthrombinAktivität im Blut 2, 4, 6, 8 und 10 Stunden nach Gabe von Menadion an Vitamin $\mathrm{K}$-Mangelrațten (Werte: $\overline{\mathrm{x}} \pm s_{x} ; \mathrm{P}$ beżogen auf Vitamin

\begin{tabular}{cccccc}
\hline Art & Zahl & $\begin{array}{c}\text { Tryptophau- } \\
\text { Pyrrolase } \\
(\mu \text { Mol Kynu- } \\
\text { renin/g } \\
\text { Protein-Std.) }\end{array}$ & $\mathbf{P}$ & $\begin{array}{c}\text { Prothrombin } \\
(\%)\end{array}$ & $\mathbf{P}$ \\
\hline Normal & 19 & $15,12 \pm 1,41$ & & $99,37 \pm 7,58$ & \\
Mangel & 27 & $12,63 \pm 1,24$ & & $3,19 \pm 0,82$ & \\
2 Stdn. & 4 & $13,38 \pm 2,57$ & $<0,4$ & $9,83 \pm 3,59$ & $<0,001$ \\
4 Stdn. & 8 & $23,94 \pm 1,14$ & $<0,001$ & $64,34 \pm 10,48$ & $<0,001$ \\
6 Stdn. & 4 & $23,44 \pm 1,89$ & $<0,001$ & $80,75 \pm 9,70$ & $<0,001$ \\
8 Stdn. & 4 & $17,08 \pm 6,19$ & $<0,005$ & $100,68 \pm 6,70$ & $<0,001$ \\
10 Stdn. & 4 & $24,87 \pm 2,93$ & $<0,001$ & $99,83 \pm 5 ; 26$ & $<0,001$ \\
\hline
\end{tabular}

Tryptophan-Pyrrolase nach der zweiten Stunde ein starker Anstieg der Aktivität. Das Maximum wurde nach der vierten Stunde erreicht, betrug das Zweifache des Ausgangswertes und lag sogar fast ein Drittel höher als bei den Normaltieren. Die Differenz der Enzymaktivität war signifikant $(P<0,001)$. Nach der vierten Stunde pendelte die Aktivität um einen Mittelwert herum, der aber immer noch höher lag' als die Kontrollwerte.

Beim Prothrombin zeigte sich der größte Anstieg $z$ wischen der zweiten und vierten Stunde, das Maximum $(100 \%)$ wurde aber erst nach der achten Stunde erreicht.

Zur Prüfung des Einflusses von Menadion auf die Aktivität der Tryptophan-Pyrrolase und des Prothrombins bei Normaltieren, wurden drei scheingespritzte Ratten mit Menadion behandelt. Die Meßwerte lagen $z$ war geringfügig höher, waren aber nicht signifikant gegenüber den unbehandelten Normaltieren verändert.

Wirkung von Menadion auf Marcumar-Vitamin K-MangelRatten in Verbindung mit den Hemmstoffen Actinomycin $D$ und Cycloheximid

Marcumar-behandelte Vitamin K-Mangelratten erhielten eine halbe Stunde vor Gabe von Menadion die Inhibitoren Actinomycin D (1 mg/kg Körpergewicht) 


\section{IIh ir Ausguß wird ein grrmer Schlucker}

Nichts von den überschüssigen Reagenzien, die sich beim Verdünnen mit Vollpipette und Meßkolben ergaben, fällt mehr für ihn $a b$.

Denn beim Brand-Dilutor brauchen Sie immer nur die Reagenzmenge, mit der Sie tatsächlich weiterarbeiten wollen. Dieser Dilutor spart aber nicht nur Reagenzien - also Geld sondern vor allem Zeit. Bei hervorragender Genauigkeit.

Mehr darüber auf der nächsten Seite
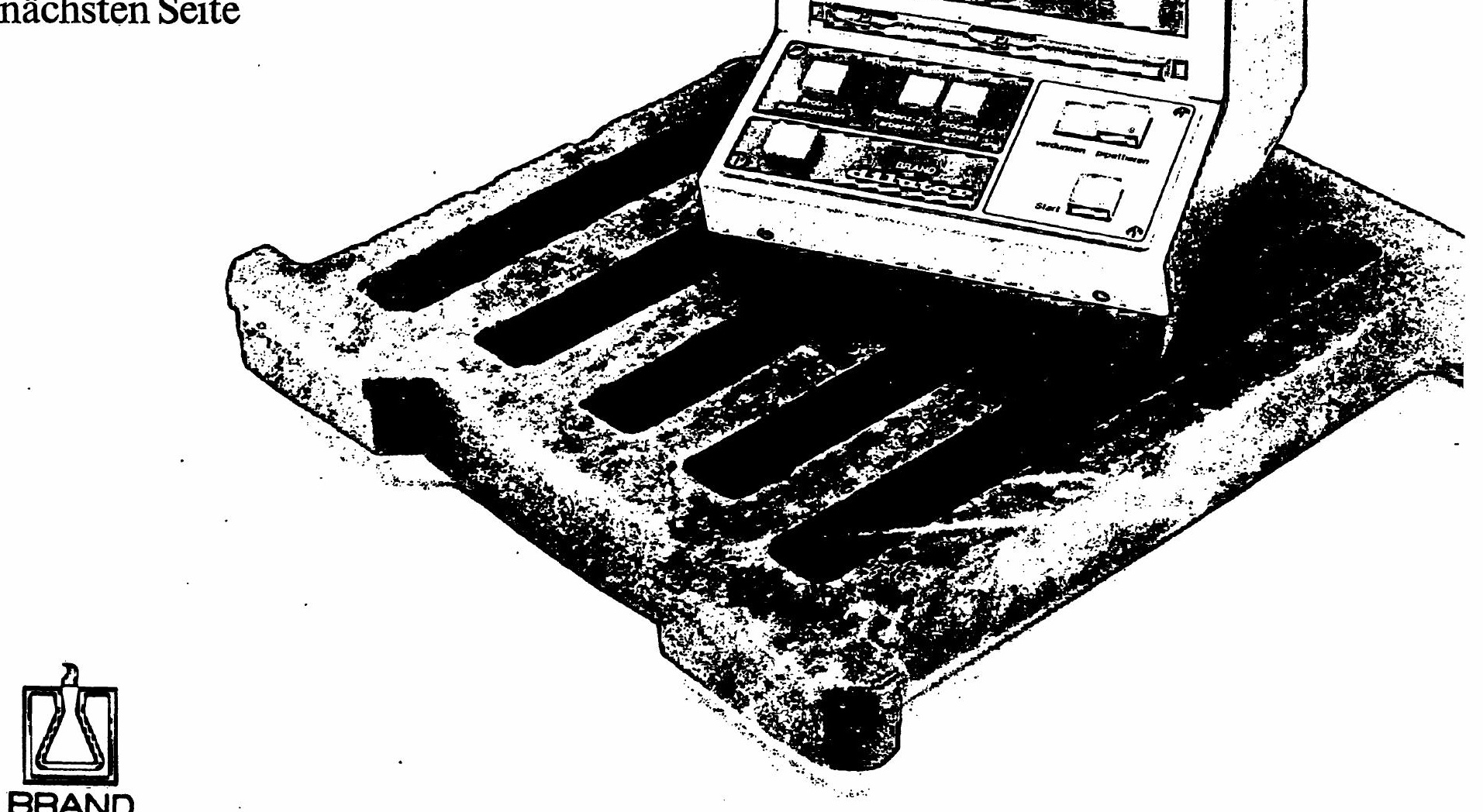

RUDOLF BRAND

LABORGERÄTE UND VAKUUMPUMPEN 698 WERTHEIM/MAIN - POSTFACH 310 


\section{BRAND-Dilutor}

Volltransistoriertes Verdünnungs- und Pipettiergerät

Strömungswege, Kolben, Zylinder und Ventil bestehen ausschließlich aus Glas und PTFE

Digitale Volumeneinstellung Reagenzzylinder: $1 \mu \mathrm{I}$ $5000 \mu l$ Probezylinder: $0,1 \mu \mathrm{l}-$ $500 \mu$ l oder: $1 \mu$ l $-5000 \mu$ l

Zweite Wähltaste ermöglicht Verwendung als Dispenser

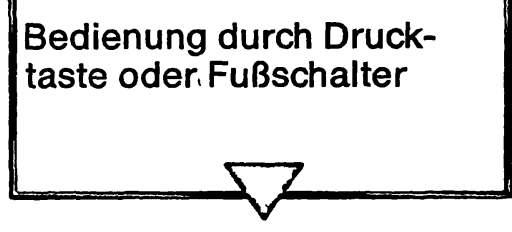

SE

Erstes elektronisch gesteuertes Verdünnungsgerät

Schnelleinstellung in Mikroschritten von $0,1 \mathrm{bzw}$. $1 \mu \mathrm{l}$ (rechnerisch 25000000 verschiedene Mischungsverhältnisse möglich)

Nur zwei bewegte Teile an jedem Zylinder

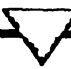

BRAND-ĠERÄTE WEIL DIE RENTABILITÄT ENTSCHEIDET

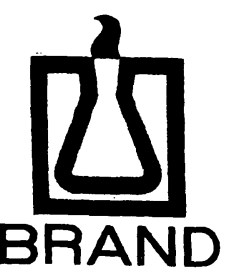

Einfache und bequeme Handhabung

Universell einsetzbar durch ausgezeichnete chemische $\checkmark$ Resistenz und Anpassung an den Verwendungsżweck

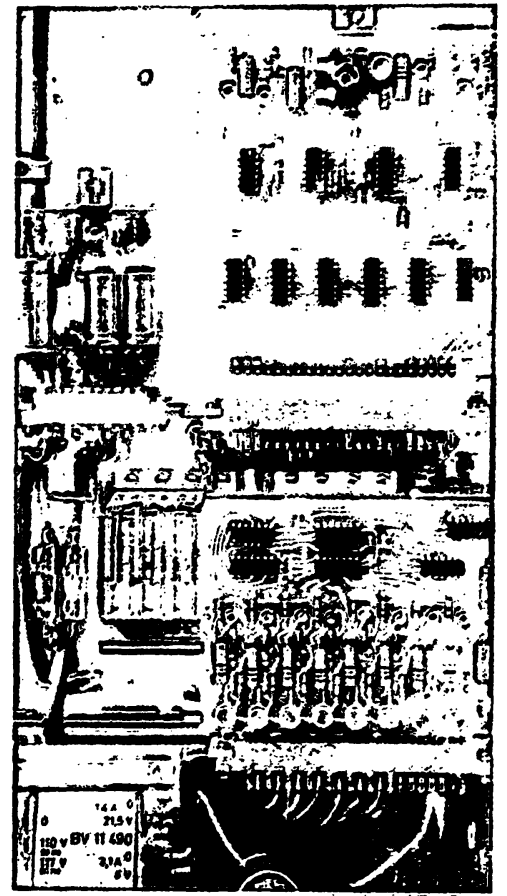

Extrem niedriger Einstell-
fehler auch bei geringen
Probevolumina

Außergewöhnlich gute Reproduzierbarkeit der Messergebnisse

\section{COUPON}

Hier konnte Ihnen natürlich nur eine Übersicht über einige wichtige Eigenschaften dieser Geräte gegeben werden. Wenn Sie aber diesen Abschnitt mit einer, notfalls unfrankierten, Postkarte an uns schicken, lasșen wir Ihnen gerne weitere informationen zukommen über:

\section{BRAN D-DILUTOR}

BRAND Fabrlk für Laborgeräte

6980 Wertheim -2 
Tab. 3

Aktivität der Tryptophan-Pyrrolase in der Leber und Prothrombin-Aktivität im Blut vier Stunden nach Gabe von Menadion an Vitamin $\mathrm{K}-$ Mangelratten, die jeweils 30 Min. vor Menadiongabe Actinomycin $\mathrm{D}$ bzw. Cycloheximid erhielten (Werte: $\overline{\mathbf{x}} \pm \mathrm{s}_{\mathbf{x}} ; \mathrm{P}$ bezogen auf Vitamin K-Mangeltiere mit Menadion nach vier Stunden)

\begin{tabular}{|c|c|c|c|c|c|c|}
\hline . & Art & Zahl & $\begin{array}{l}\text { Tryptophan-Pyrrolase } \\
\text { ( } \mu \text { Mol Kynurenin/g } \\
\text { Protein - Std.) }\end{array}$ & $\mathbf{P}$ & Prothrombin (\%) & $P$ \\
\hline & Mangel + Menation & 8 & $23,94 \pm 1,14$ & & $64,34 \pm 10,48$ & \\
\hline & $\begin{array}{c}\text { Mangel }+ \text { Actino- } \\
\text { mycin }+ \text { Menadion }\end{array}$ & 8 & $9,84 \pm 2,09$ & $<0,001$ & $3,36 \pm 1,51$ & $<0,001$ \\
\hline & $\begin{array}{l}\text { Mangel }+ \text { Cyclo- } \\
\text { heximid }+ \text { Menadion }\end{array}$ & 9 & $6,75 \pm 0,37$ & $<0,001$ & $2,52 \pm 0,34$ & $<0,001$ \\
\hline
\end{tabular}

oder Cycloheximid (10 mg/kg Körpergewicht) intraperitoneal gespritzt. Nach vier Stdn. erfolgte die Messung des Gesamteiweißes, der Tryptophan-Pyrrolase und des Prothrombins. Kontrolltiere waren Mangeltiere, die keine Hemmstoffe erhielten.

In Tabelle 3 werden die Enzymaktivitäten der Vitamin K-Mangeltiere, die $30 \mathrm{Min}$. vor der Menadionbehandlung Actinomycin D oder Cycloheximid erhielten, und der Kontrolltiere verglichen. Actinomycin D und Cycloheximid verhinderten den Aktivitätsanstieg der Tryptophan-Pyrrolase und des Prothrombins. Es zeigte sich sogar ein weiterer Abfall: Die Tryptophan-Pyrrolase sank nach Vorbehandlung mit Actinomycin D um etwa 20\% und nach Cycloheximid um fast 50\% weiter ab. Der Prothrombinspiegel blieb bei Actinomycin D unverändert niedrig, fiel aber bei Cycloheximid noch um fast ein Drittel ab. Somit läßt sich der Aktivitätsanstieg der Tryptophan-Pyrrolase und des Prothrombins durch vorgeschaltete Hemmung der Proteinsynthese unterbinden.

Höhere Dosen von Cycloheximid $(20 \mathrm{mg} / \mathrm{kg}$ Körpergewicht) ergeben zwar einen stärkeren Abfall der Enzymaktivität, waren aber nicht statistisch auszuwerten, da die meisten Tiere schon nach drei bis vier Stdn. starben. Der Qurck-Wert lag unter 1\%.

\section{Diskussion}

In den vorliegenden Untersuchungen wurde der Einfluß von Vitamin $K_{3}$ auf die Aktivität verschiedener Gerinnungsfaktoren im Blut (II, V) und Enzyme in der Leber mit kurzer Halbwertszeit (Tryptophan-Pyrrolase, Serin-Dehydratase) verglichen mit der Aktivität eines Enzyms mit langer Halbwertszeit (Aldolase) (27). Die Halbwertszeit der Faktoren II und $\mathrm{V}$ wird mit 1-3 Stdn. (12-14), der Tryptophan-Pyrrolase mit 2-3 Stdn. (28-31) und der Serin-Dehydratase mit 4-20 Stdn. (32) in der Rattenleber angegeben. Das Ziel der Untersuchungen war es zu zeigen, ob unter Vitamin K-Mangel nur die Gerinnungsfaktoren II und $\mathrm{V}$ oder auch andere Proteine mit kurzer Halbwertszeit in ihrer Aktivität bzw. in ihrer Synthese vermindert sind. Mit diesen Untersuchungen war zugleich die Frage nach der Spezifität der Wirkung von Vitamin $\mathrm{K}$ verbunden.

Die Enzyme Tryptophan-Pyrrolase und Serin-Dehydratase sind adaptative Enzyme (18, 21, 33-35), d. h. ihre Aktivität ist in starkem Maße von der Nahrungszufuhr abhängig. Daher wurden die vorliegenden
Untersuchungen ausnahmslos an Hungertieren vorgenommen. Hierdurch war die Streurate der Meßwerte deutlich vermindert.

Der Vitamin K-Mangelzustand, der in dieser Arbeit durch Injektion von Marcumar erzeugt wurde, ist von den alimentären Vitamin K-Mangelzuständen zu unterscheiden. Es muß daran gedacht werden, daß Marcumar durch Entkopplung der oxydativen Phosphorylierung die Synthese der Proteine allgemein hemmt, was für den alimentären Vitamin K-Mangel in dieser direkten Form nicht zutreffen muß (36-40).

In Marcumar-behandelten Tieren war die Aktivität der Tryptophan-Pyrrolase und Serin-Dehydratase in der Leber parallel mit den Gerinnungsfaktoren II und V im Blut vermindert. Im Gegensatz dazu war der Aktivitätsabfall der Aldolase wegen ihrer langen Halbwertszeit unter unseren Versuchsbedingungen nur gering und nicht signifikant. Diese Befunde können als generelle Hemmung der Proteinsynthese infolge Vitamin K-Mangel gedeutet werden, wobei der Wirkungsmechanismus und der Wirkungsort nach unklar ist. $\mathrm{Zu}$ diskutieren wäre hier eine unspezifische Beeinflussung der Proteinsynthese auf der Höhe der Transscription $(7,8)$, oder am Ribosom bei der Translation $(5,9,10)$, bzw. bei der Ablösung des Proteins vom Ribosom (11). Weiterhin wäre èine verminderte Energiebereitstellung möglich (41). Eine Stabilisierung von Enzymen und Gerinnungsfaktoren durch Vitamin K ist unwahrscheinlich.

Aufschlußreich sind die Befunde an den Gerinnungsfaktoren und Enzymen nach Vitamin K-Gabe. Injiziert man Hungertieren Vitamin $K_{3}$, so steigt die $A k-$ tivität der Tryptophan-Pyrrolase nur geringgradig und nicht signifikant an. Verabreicht man aber Vitamin $K_{3}$ an Vitamin K-Mangeltiere, so steigt die Aktivität der untersuchten Enzyme über den Kontrollwert hinaus an, wobei maximale Aktivitäten nach etwa vier Stdn. erreicht werden.

Zur Frage der Ursache des Enzymaktivitätsanstiegs bzw. der Zunahme der Aktivität des Gerinnungsfaktors II wurden Proteinsynthesehemmer (42-44) eingesetzt. Actinomycin $\mathrm{D}$ hemmt in einer Konzentration von $1 \mathrm{mg} / \mathrm{kg}$ Körpergewicht den Anstieg der Tryptophan-Pyrrolase-Aktivität bei Menadiongabe an Vitamin K-Mangeltiere. Der Effekt von Cycloheximid $(10 \mathrm{mg} / \mathrm{kg}$ Körpergewicht) ist noch ausgeprägter. Bemerkenswert ist, daß sowohl Actinomycin D als auch Cycloheximid den Aktivitätsanstieg verhindern. Somit 
läßt sich vermuten, daß der Anstieg der TryptophanPyrrolase-Aktivität und der Aktivität des Gerinnungsfaktors II auf einer de novo Proteinsynthese beruht, wie dies für Prothrombin auf immunologischem Wege bewiesen worden ist (5).

Die Wirkung von Actinomycin $\mathrm{D}$ in der verabfolgten Dosis (1 mg/kg Körpergewicht) ist vieldeutig. Da Vitamin $\mathrm{K}$ in den Mangeltieren die Aktivität der Gerinnungsfaktoren und Enzyme offenbar unspezifisch steigert und Cycloheximid diesen Aktivitätsanstieg unterbindet, könnte der Wirkort von Vitamin $\mathrm{K}$ an den Ribosomen liegen. Untersuchungen des ATPGehaltes in der Leber nach Marcumargabe liegen nicht vor. Somit ist zu vermuten, daß entweder die Energie- bereitstellung oder die Proteinsynthese am Ribosom auf eine unspezifische Weise aktiviert wird.

Das von Ouson und Mitarbeitern $(10,6,5)$ postulierte regulatorische Protein hätte demnach eine unspezifische Wirkung auf die ribosomale Proteinsynthese.

Die verminderte Aktivität der Tryptophan-Pyrrolase und Serin-Dehydratase und der Gerinnungsfaktoren unter.Marcumarwirkung hat therapeutische Bedeutung. Unsere Befunde deuten darauf hin, daß bei Patienten mit langer Marcumarbehandlung nicht nur der Prothrombinspiegel vermindert ist, sondern auch Enzyme in ihrer Aktivität verändert und hierdurch nicht beabsichtigte Stoffwechselstörungen, z. B. im Aminosäurestoffwechsel, hervorgerufen werden können.

\section{Literatur}

1. DAM, H. und F. SCHÖNHEYDER, J. Biochem. 28, 1355 (1934). 2. Quick, A. J., Amer. J. Physiol 118, 260 (1937). - 3. Martir, R., J. L. Ambrus, J. E. Sokal und I. Mink, Proc. Soc. exper. Biol. Med. 116, 69 (1964). - 4. Otson, J. P., J. Clin. Invest. 45, 690 (1966). - 5. Li, L. F., R. K. Kipfer und R. E. Olson, Arch. Biochem. Biophysics 137, 494 (1970). - 6. Orson, R. E., R. K. Kipfer und L. F. Li, Adv. Enzym. Reg. 7, 83 (1969). - 7. Olson, R. E., Science 145, 926 (1964). - 8. Olson, R. E., Canad. J. Biochem. 43, 1565 (1965). - 9. Johnson, B. C., R. B. HiLl, R. Alden und G. S. Ranhotra, Life Sci. 5, 385 (1966). - 10. Olson, R. E., G. R. Philipps und N. T. Wang, Adv. Enzym. Reg. 6, 213 (1968). - 11. Hill, R. B., S. Gaetani, A. M. Paoluccr, P. B. Rama-Rao, R. Alden, G. S. Ranhotra, D. V. Shah, V. K. Shaf und B. C. Johnson, J. biol. Chemistry 243, 3930 (1968). 12. Wright, I. S., F. Koller und F. Streuli, Thromb. Diathes. Haemorrh. Suppl. 4, 101 (1960). - 13. Harper, P. V., E. Calvary und J. G. Allen, Fed. Proc. 15, 87 (1956). - 14. Stefanini, M. und V. A. Pisciorta, Science 111, 364 (1950). - 15. Geschwind, I. I. und C. H. Li, Nature 172, 732 (1935). - 16. Geschwind, I. I. und C. H. Lr, J. Clin. Endocr., Springfield 14, 789 (1954). 17. KNox, W. E. und V. H. Auerbach, J. biol. Chemistry 214, 307 (1955). - 18. KNox, W. E., Brit. J. exper. Path. 32, 462 (1951). - 19. Fallon, H. J., E. J. Hackney und W. L. Byrne, J. biol. Chemistry 241, 4157 (1966). - 20. Drews, J., Ptoc. Soc. exper. Biol. Med. 126, 671 (1967). - 21. Feigelson, P. und O. Greengard, Adv. Enzym. Reg. 7, 119 (1968). - 22. Hartert, H. und G. LeUbE, Diagnostik 3, 242 (1970). - 23. KINGSLEY, G. R., J. biol. Chemistry 131, 197 (1939). - 24. BRUNs, F. H. und H. U. Bergareyer, in H. U. Bergmeyer: Methoden der enzymat.
Analyse. Verlag Chemie, Weinheim/Bergstr. S. 724 (1962). 25. Prtot, H. C. und N. Pries, Analytic. Biochem. 9, 454 (1964). 26. Periano, C., J. biol. Chemistry 242, 3860 (1967). - 27. Schapira, G., J. KRUH, J. C. Drẹyfus und F. Schapira, J. biol. Chemistry 235, 1738 (1960). - 28. SCHIMKe, R. T., E. W. SwEENEY und C. M. Berlin, J. biol. Chemistry 240, 322 (1965). - 29. Knox, W. E. und M. M. Prras, J. biol. Chemistry 242, 2959 (1967). - 30. Cho-Chung, Y. S. und H. C. Prtrot, Europ. J. Biochem. 3, 401 (1968). - 31. LABRI, F. und A. KorNER, J. biol. Chemistry 243, 1116 (1968). - 32. Jost, J. P., E. A. KHAIRALLAH und H. C. Prtor, J. biol. Chemistry 243, 3057 (1968). - 33. Knox, W. E. und A. H. Merler, Science 113, 237 (1951). 34. Wood, J. S. und W. E. Knox, Proc. Amer. Ass. Canc. Res. 45th Meet. 1, 52 (1954). - 35. CAFFERY, J. M., L. Wichard und J. L. IRvin, Biochim. biophysica Acta, Amsterdam 157, 616 (1968). - 36. Marrius, C. und D. Nitz-Litzow, Biochim. biophysica Acta, Amsterdam 12, 134 (1953). - 37. Martrus, C. und D. Nrrz-Lrrzow, Biochim. biophysica Acta, Amsterdam 13, 152 (1954). - 38. Martius, C. und D. Nrtz-Litzow, Biochim. biophysica Acta, Amsterdam 13, 288 (1954). - 39. Parmar, S. S. und J. Löwenthat, Biochem. Biophys. Res. Com. 8, 107 (1962). 40. Berry, D., E. Berry und R. E. Olson, Fed. Proc. 25, 542 (1966). - 41. Martius, C., Dtsch. med. Wschr. 83, 1701 (1958). 42. Trakatellis, A.' C., A. E. Axelrod und M. Montjar, Nature 203, 1134 (1964). - 43. Wetrstern, F. O., H. Noll und S. Penman, Biochim. biophysica Acta, Amsterdam 87, 525. 44. Colombo, B., L. Felicetri und C. Bagliont, Biochem. Biophys. Res. Com. 18, 389 (1965).
Priv.-Doz. Dr. H. Reinauer 4000 Düsseldorf 1 Witzelstr. 111 